ovarian serous carcinoma in specialized care center in Montréal (Québec, CANADA) from 1999 to 2019. Neighborhood socioeconomic status at diagnosis for each woman was inferred from their postal code area linked to socioeconomic indicators derived from the Canadian census data. Immunological age was measured using a forensic medicine validated blood biomarker (TRECs - T-cell receptor excision circles). Blood sample were available before treatments $(\mathrm{n}=196)$, after treatments $(\mathrm{n}=270)$, or paired before and after treatments $(n=22)$. Time was calculated from diagnosis date to death, relapse or study censoring (last relapse-free follow-up date).

Result(s)* In our study sample, neighborhood socioeconomic status at diagnosis did not affect overall survival. We observed that accelerated immunological aging was occurring before treatment initiation $(\mathrm{n}=218, \mathrm{p}<0.0001)$. Treatment itself was not associated with a significant additional accelerated immunoaging $(n=22)$. Neither higher FIGO stage at diagnosis nor neighborhood socioeconomic status at diagnosis were associated with a higher disparity between chronological and immunological age.

Conclusion* Our preliminary study results support that either cancer itself causes a significant immunological age shift in women suffering from a primary ovarian serous carcinoma, or that women suffering from accelerated aging are more prone to develop cancer. Although statistical underpower and a biased sample effect cannot be excluded, the socioeconomic status does not seem to influence accelerated immunoaging nor overall survival.

\section{CLINICAL MANAGEMENT AND OUTCOMES OF PRIMARY OVARIAN SARCOMAS - A SINGLE CENTER EXPERIENCE}

V Theodoulidis*, DE Vlachos, C Theofanakis, V Pergialiotis, N Thomakos, A Rodolakis, D Haidopoulos. National Univesity of Athens, First Department of Obstetrics and Gynecology, Athens, Greece

\subsection{6/ijgc-2021-ESG0.485}

Introduction/Background* Primary ovarian sarcoma is a very rare malignancy representing $<2 \%$ of ovarian cancer. Aim of this study is to investigate the clinicopathologic features and outcomes in patients with primary sarcoma of the ovary.

Methodology We retrospectively reviewed between 2012 and 2019, all patients with primary ovarian sarcoma who were treated at Alexandra Hospital of Athens.

Result(s)* A total of 17 patients with ovarian sarcoma had been surgically treated. The median age of patients was 61 [33-84] years. The most common symptom was abdominal pain and bloating. The majority of patients were postmenopausal (14) and only three were premenopausal. The histopathological diagnosis revealed 14 patients with carcinosarcoma, two with ovarian endometrial stromal sarcoma and one with leiomyosarcoma. After the surgery 23\% of patients were at stage I, $12 \%$ at stage II, $53 \%$ at stage III and $12 \%$ at stage IV. Only $5(29 \%)$ patients had suboptimal debulking surgery and 12 (71\%) had optimal debulking surgery. The mean number of chemotherapy courses were $6.8 \pm 4$ with unsatisfactory response. Five patients had a recurrence at two years and 4 of them had a secondary debulking surgery.

Conclusion* Primary ovarian sarcoma has a poor prognosis. Optimal debulking surgery appears to be a significant factor for recurrence. There is a need for more studies to explore the role of optimal debulking surgery and adjuvant chemotherapy in primary ovarian sarcoma.

\section{TREATMENT STRATEGIES, DISEASE RECURRENCE AND SURVIVAL OF WOMEN WITH MALIGNANT SEX CORD- STROMAL CELL TUMOURS IN GERMANY}

${ }^{1} \mathrm{~A}$ Hasenburg*, ${ }^{2} \mathrm{H}$ Plett, ${ }^{2} \mathrm{P}$ Harter, ${ }^{2} \mathrm{~F}$ Heitz, ${ }^{3} \mathrm{~S}$ Kommoss, ${ }^{4} \mathrm{~J}$ Keul, ${ }^{5} \mathrm{E}$ Roser, ${ }^{5} \mathrm{~J}$ Sehoulli ${ }^{5}$ E loana Braicu, ${ }^{6} \mathrm{M}$ Bossart, ${ }^{6} \mathrm{MF}$ Hasanov, ${ }^{7} \mathrm{~B}$ Czogalla, ${ }^{7} \mathrm{~A}$ Burges, ${ }^{8} \mathrm{~T}$ Link, ${ }^{9} \mathrm{M}$ Doris, ${ }^{10} \mathrm{~A}$ Staebler, ${ }^{11} \mathrm{~L}$ Hanker, ${ }^{6} \mathrm{M}$ Klar. ${ }^{1}$ University Centre Mainz, Faculty of Medicine Mainz, Obstetrics and Gynaecology, Mainz; ${ }^{2}$ Evang. Huyssens-Stiftung Essen-Huttrop (eine Einrichtung der KEM | Evang. Kliniken Essen-Mitte gGmbH), Essen, Germany; ${ }^{3} T u e b i n g e n$ University Hospital, Department of Women's Health, Tuebingen, Germany; ${ }^{4}$ University Hospital Tuebingen, Obstetrics and Gynaecology, Tuebingen, Germany; ${ }^{5}$ Charité Universitätsmedizin Berlin, Department of Gynecology with Center for Oncological Surgery, Berlin, Germany; ${ }^{6}$ University Hospital of Freiburg, Obstetrics and Gynaecology, Freiburg, Germany; 'University Hospital, LMU Munich, Obstetrics and Gynaecology, Munich, Germany; ${ }^{8}$ Universitätsklinikum Carl Gustav Carus Dresden; TU Dresden, Obstetrics and Gynaecology, Dresden, Germany; ${ }^{9}$ University Hospital, LMU Munich, Institute of Pathology, Munich, Germany; ${ }^{10}$ University Hospital of Tuebingen, Institute of Pathology, Germany; ${ }^{11}$ University Hospital Schleswig-Holstein, Luebeck, Obstetrics and Gynaecology, Luebeck, Germany

\subsection{6/ijgc-2021-ESG0.486}

Introduction/Background* Malignant sex cord-stromal cell tumours (SCST) account for about $7 \%$ of all ovarian malignancies and higher-level evidence on these tumours is scarce. The Arbeitsgemeinschaft fuer Gynaekologische Onkologie (AGO) has established a clinico-pathological database (Current Ovarian geRm cell and SEx cord stromal Tumour Treatment strategies, CORSETT) for this rare disease.

Methodology 20 German centres entered mixed retro- and prospective data of SCST patients treated between 2000 to 2014 into the CORSETT database. Descriptive analyses of the treatment strategies, Kaplan-Meier curves and cox regression analyses for the survival analysis were conducted. Tumour reference pathology was provided.

Result(s)* 235 patients with granulosa cell tumour (GCT, median age 52 years) and 24 patients with sertoli-leydig cell tumour (SLCT, median age 33.5 years) were included. Main surgical treatment strategy was laparoscopy (50.9\%) and fertility-sparing surgery was performed in 105 GCT (54.9\%) and in $14(58.3 \%)$ SLCT patients. In 38 GCT (23.6\%) and two $(9.25 \%)$ SLCT patients, the tumour ruptured intra-operatively. 44 GCT $(18.7 \%)$ and two (8.3\%) SLCT patients received adjuvant systemic treatment (most common regimen: paclitaxel/carboplatinum, 52.3\%). Median PFS was 80.4 months (95\% CI 67.8 - 99.6) in GCT and was not reached in SLCT patients. 105 GCT patients (44.5\%) experienced disease recurrence. Advanced FIGO stage $(\mathrm{p}=0.04)$ and intra-operative cyst rupture $(\mathrm{p}=0.02)$ was associated with disease recurrence.

Conclusion* Fertility-sparing surgery was offered in most SCST patients in Germany over the past years, and intra-operative tumour rupture occurred in almost every fourth GCT patient. The most commonly applied adjuvant chemotherapy regimen was taxane and carboplatinum, which had however no effect on disease recurrence. Despite excellent overall survival rates in GCT and SLCT patients, recurrence rate in GCT patients was high. 